Unfallchirurg 2010 $113: 75$

DOI 10.1007/s00113-009-1739-2

Online publiziert: 13. Januar 2010

(c) Springer-Verlag 2010

Redaktion

W. Mutschler, München
P. Kalbe ${ }^{1,2}$

${ }^{1}$ Deutsche Gesellschaft für Unfallchirurgie (DGU), Berlin

${ }^{2}$ Chirurgisch-orthopädische Gemeinschaftspraxis Dr. Kalbe \& Dr. Kant, Rinteln

\section{Ein Menetekel}

\section{Kommentar zum Beitrag}

Lindhorst $E$, Hoffmann $R$, von Seebach $M$ et al (2009) "Röntgendiagnostik - fachgebunden Skelett" und "Magnetresonanztomographie fachgebunden". Versuch einer Analyse des Erreichten und Erreichbaren. Unfallchirurg 112. doi: s00113-009-1699-6

Die Röntgendiagnostik des Skeletts („Teilradiologie“) ist ein unverzichtbares Kernstück der Tätigkeit des Unfallchirurgen und Orthopäden. An dieser Aussage dürfte es unter Unfallchirurgen keinen Zweifel geben. Gleichwohl ist mit der letzten Neuordnung der Weiterbildungsordnung (WBO) 2003 dieser wichtige Teil unserer täglichen Arbeit aus der Definition des Facharztes für Orthopädie und Unfallchirurgie herausgeschnitten und in eine Zusatzweiterbildung verlagert worden. Da sich die Umsetzung der WBO in den Landesärztekammern lange hingezogen hat, wird die Brisanz dieser Entscheidung erst jetzt langsam deutlich. Die Fachärzte für Orthopädie und Unfallchirurgie (FAOU) nach der neuen WBO haben keinen formalen Nachweis der Weiterbildung in der diagnostischen Skelettradiologie. Wenn diese Kollegen leitende Positionen besetzen, wird es absehbar in naher Zukunft Probleme mit der Vermittlung dieser Weiterbildungsinhalte geben.

Die Autoren bringen in ihrem Beitrag in diesem Heft deutlich zum Ausdruck, dass dies zum jetzigen Zeitpunkt noch keine Auswirkungen auf die praktische Tätigkeit in der Klinik und Praxis hat. Bestehende Betriebs- und Abrechnungsgenehmigungen für die Röntgendiagnostik werden (noch) nicht tangiert, sofern die Fachkunde im Strahlenschutz gemäß der Röntgenverordnung nachgewiesen werden kann. Die Bedingungen könnten sich jedoch in der Zukunft ändern. Schon jetzt ist der Zugang zur Abrechnung von Röntgenleistungen für den FAOU nach der neuen WBO deutlich schwieriger und unsicherer. Daher können wir es auf die Dauer nicht hinnehmen, dass ein unverzichtbarer Anteil unserer unfallchirurgischen Tätigkeit nicht in der Weiterbildung enthalten ist. Der Berufsständische Ausschuss (BSA) und der Ausschuss Vertretung niedergelassener Vertragsärzte (ANV) der DGU haben daher gemeinsam mit den Orthopäden über die DGCH und den BDC einen Antrag an die Ständige Kommission Weiterbildung (Stäko) der Bundesärztekammer unterstützt, die Skelettradiologie und in Zukunft auch die MRT-Diagnostik in die Weiterbildung zum FAOU einzubinden. Leider fand sich hiervon nichts in den vorläufigen Planungen für Änderungsanträge zur Muster-WBO für den 113. Ärztetag in Dresden im Jahr 2010 wieder. Dies kann so nicht hingenommen werden. Durch regionale Initiativen wird nunmehr ein alternativer Weg aus diesem Dilemma gesucht. In Berlin gibt es einen entsprechenden Beschluss der Delegiertenversammlung der Ärztekammer, der allerdings noch nicht rechtskräftig ist. Alle Mitglieder der DGU und der DGOU sollten über ihre Landesärztekammern unser Anliegen argumentativ unterstützen.

Wenn die leitenden und niedergelassenen Unfallchirurgen jetzt nicht vehement die Kompetenz für die radiologische Diagnostik des Skeletts beanspruchen, kann in Zukunft auch keine entsprechende Weiterbildungsbefugnis reklamiert werden. Wir würden so die Chance verspielen, die wesentlichen Inhalte unseres Fachs aus eigener Kompetenz weiterzubilden und so unseren eigenen Nachwuchs zu generieren. Die Empfehlung, die Zusatzweiterbildung „Diagnostische Radiologie: Skelett“ nach den Übergangsbestimmungen möglichst noch zu erwerben, ist eine pragmatische Zwischenlösung und wird von unseren Fachgesellschaften unterstützt. Auf die Dauer können wir uns aber nicht mit der Beschränkung auf die konventionelle Skelettradiologie zufrieden geben. Die selbst durchgeführte Bildgebung mit Röntgen, Sonographie und letztendlich auch MRT ist für uns ebenso wichtig wie die Benutzung chirurgischer Instrumente. Weder können wir unsere Patienten jederzeit zum Facharzt für Radiologie schicken noch können die Radiologen in unseren OPs für Durchleuchtungen, Navigationen, intraoperative $2 \mathrm{D}$ - oder $3 \mathrm{D}$-Scans zur Verfügung stehen. Die knappen Ressourcen des Gesundheitswesens werden dies auch in Zukunft nicht gestatten.

Jeder Unfallchirurg und Orthopäde kann anhand der Tabelle im Beitrag von Lindhorst et al. überprüfen, ob für ihn aktuell Handlungsbedarf besteht. Besonders auf die Erneuerung der Fachkunde im Strahlenschutz alle 5 Jahre muss geachtet werden, da ansonsten ein neuer Grundkurs erforderlich wird.

\section{Korrespondenzadresse \\ Dr. P. Kalbe}

Chirurgisch-orthopädische Gemeinschaftspraxis Dr. Kalbe \& Dr. Kant

Josua-Stegmann-Wall 7, 31737 Rinteln

kalbe@bdc.de

Der Autor ist Vorsitzender des Ausschusses Vertretung niedergelassener Vertragsärzte und Fachbeirat für niedergelassene Unfallchirurgen. 